\title{
Classification of Molecular Biomarkers
}

\author{
Ankeet Shah, Dominic C. Grimberg, Brant A. Inman ${ }^{凶}$ \\ Duke Cancer Institute, Division of Urology, Duke University Medical Center, Durham, United States
}

\begin{abstract}
A "biomarker" is any measurable characteristic that indicates the presence or absence of disease or the biological response to a stimulus, typically an exposure or intervention. The FDA-NIH Biomarker Working Group has produced a document called Biomarkers, EndpointS and other Tools (BEST), which defines 7 categories of biomarkers according to their clinical usage: susceptibility and risk, diagnostic, monitoring, prognostic, predictive, pharmacodynamic and treatment response, and safety. We approach the classification of biomarkers in 2 additional ways: their bodily source and their measurement type. In the context of their use in genitourinary malignancy, we also consider factors that influence their use and reliability in clinical and research applications.
\end{abstract}

\section{Introduction}

A "biomarker" is any measurable characteristic that indicates the presence or absence of disease or the biological response to a stimulus, typically an exposure or intervention. The FDA-NIH Biomarker Working Group has defined 7 categories of biomarkers according to their clinical usage: susceptibility and risk, diagnostic, monitoring, prognostic, predictive, pharmacodynamic and treatment response, and safety. We approach the classification of biomarkers in 2 additional ways: their bodily source and their measurement type. In the context of their use in genitourinary malignancy, we also consider factors that influence their use and reliability in clinical and research applications.

\section{Biomarkers by Source}

\section{Blood}

Blood and its various components represent a valuable source for a wide variety of molecular biomarkers. Although direct sampling of cells in solid tumours of urologic oncology is not accomplished with peripheral blood draws, circulating tumour cells, as well as cell-free circulating DNA, can be used for genomic biomarkers [1,2]. Proteomics, lipidomics, and metabolomics in oncology are growing fields that can also be applied to blood samples for additional biomarker evaluation [3].

The means used to obtain blood are less invasive than those used to obtain tissue and some biofluids, and many patients with urologic malignancies are likely to undergo blood draws for standard care. Blood is largely composed of water but also contains erythrocytes, leukocytes, platelets, fibrinogen and other clotting factors, proteins including albumins and globulins, glucose, and electrolytes. Importantly, these components may limit the assessment of a given analyte if the blood is not processed appropriately [4,5]. It is also challenging to control the variation of individual components that make up blood that can occur in disease states such as dehydration, infection, or malignancy $[3,4,6]$.

To prevent degradation, blood and blood fractions have traditionally been cryopreserved in aliquots to limit the damage to target analytes caused by thawing and re-freezing within the specimen. A major critique of this approach is that the cost associated with cryopreservation can be significant $[7,8]$. Alternative methods of storage that aim to decrease costs tied to cryopreservation include drying with newer methods such as lyophilization and isothermal vitrification; however, these methods are not yet standardized $[9,10]$. For low molecular-weight protein, drying on silica

\section{Key Words}

Biomarker, nucleic acids, cryopreservation, tissue fixation, body fluids, tissues, urologic oncology

\section{Competing Interests}

None declared

\section{Article Information}

Received on June 30, 2020

Accepted on August 1, 2020

Soc Int Urol J. 2020; 1(1):8-15 


\section{Abbreviations}

DNA deoxyribonucleic acid

DNase deoxyribonuclease

FFPE formalin-fixed, paraffin-embedded

RNA ribonucleic acid

RNase ribonuclease

chips is feasible but does not protect specimens at higher temperatures. Dried blood spots using a paper system to evaporate water and contain blood components are useful in settings where access to cooling is limited for initial specimen handling. However, DBS requires controlled storage conditions, and certain analytes are more susceptible to oxidative damage. Novel techniques for safeguarding blood components remain an area of exploration [10].

\section{Serum and plasma}

Although whole blood has many uses for biomarker assessment, certain measurement modalities require sample refinement to optimize detection of a particular analyte. To this end, separating the cellular fraction out from the liquid portion of blood facilitates spectroscopybased analysis with less interference from blood cells. The liquid fraction of blood can be isolated as either serum or plasma. Plasma is stored in a way that prevents coagulation and clot formation. Various clotting factors, fibrinogen, and platelets are maintained in suspension in plasma. Serum, on the other hand, is allowed to clot over 30 minutes before use and can give a cleaner sample when interference from platelets and other contaminants is undesirable. There are trade-offs of the 2 forms $[4,11]$, and the liquid fraction used should be individualized to the analyte of interest [12].

\section{Cellular fractions}

Cellular components of blood are also used in a variety of biomarkers. For example, a high neutrophil to lymphocyte ratio has been found to be a poor prognostic marker of systemic inflammation and to correspond to worse outcomes in a variety of malignancies [13,14], while anemia and thrombocytopenia are used in risk stratification for renal cell carcinoma [15] and may broadly correlate with late stage tumours [16]. Isolation of cellular fractions may be achieved by centrifugation and separation by size or using advanced spectroscopy $[17,18]$. Cellular fractions are less subject to coagulation when blood is stored as plasma. Reassessment of cellular biomarkers from blood samples may be facilitated with such specimens, although the anticoagulant or freezing technique used may affect the viability of cells $[19,20]$. Flow cytometry and other immunological techniques can be used to characterize the cellular components of blood to a high degree of precision using fluorescent antibody labelling [21].

\section{Urine}

Among the least invasive liquid biomarkers to obtain, urine also has the advantage of a simpler constituent matrix than other biofluids. Urine is more thermodynamically stable than other biofluids and generally requires less processing for preservation. Also, in the case of urinary tract facing malignancies, an opportunity exists to capture tumour cells and their biochemical by-products. Urinary extracellular vesicles containing a wide variety of molecular biomarker classes have also been discovered. A vast majority of the molecular biomarker classes are identifiable in urine. Not all patients are able to supply urine for analysis, depending on their renal function or disease state. When urine can be provided, it is subject to variations in composition and $\mathrm{pH}$, which can have varying effects on any given class of biomarker. Uniquely, urine is also subject to contamination by the urinary microbiome, which can make interpretation of the source of particular analytes challenging [22-25].

\section{Ejaculate and Prostatic Secretions}

Of particular relevance to prostate cancer are prostatic biofluids, which capture analytes more effectively than other sources [26]. Of course, an intact prostate and ejaculatory pathway is required for procuring these specimens. The post-prostatic massage urine is a proxy for capturing prostatic secretions, and so this particular biofluid is also subject to the constraints of urinary specimens noted above. There are different social acceptability thresholds for semen and prostatic secretions, compared to other biofluids, making these secretions more procedurally intensive to collect. Recent efforts have shown the ability to collect RNA, DNA, proteins, and other molecular biomarkers from these biofluids [26-30]. Few data exist on storage considerations of prostatic secretions, although cryopreservation of seminal ejaculate is a standard practice in fertility scenarios $[2,27,30]$.

\section{Tissue}

Arguably, tissue is the most invasive specimen type to obtain, and using tissue has additional costs for procurement, processing, and storage. In urologic oncology, though, tissue samples are often already obtained during routine clinical practice and may be used to identify biomarkers that guide treatment or provide prognostic information [31,32]. The full range of molecular biomarkers can be obtained from tissue samples, including more direct measurement of immune parameters at the tumour site (eg, tumourinfiltrating leukocytes), which influences endogenous 
immune response to tumour as well as chemotherapy and immunotherapy efficacy $[33,34]$.

A major advantage of tissue specimens is the inherent ease with which the signal-to-noise ratio can be optimized in evaluating molecular biomarkers derived from tumours or tumour microenvironments. Depending on the biomarker of interest, a sample may be "enriched" to exclude normal tissue and prioritize tumour tissue for analysis (eg, laser capture microdissection). Recently, efforts have been made to standardize the manner in which tissue samples for various types of tumours, are delineated from surrounding stroma on histopathologic analysis with the intent of decreasing inter-observer variability of certain biomarker assessments [35].

Like other sources of biomarkers, tissuebased biomarkers are subject to degradation and contamination. This is particularly true in fresh frozen tissue samples, in which tissue will be subject to predictable ischemic changes in the ex vivo state, such as apoptosis and in situ coagulation until freezing occurs. The timeliness of such processing would affect the accuracy and quality of biomarker analysis across a range of analytes, including more sensitive proteins [36].

Formalin-fixed, paraffin-embedded (FFPE) samples increase the longevity of the specimen regardless of storage temperature. However, residual paraffin (even after appropriate treatment) can contaminate the analysis of such a preserved sample [36]. There are tradeoffs of additional processing considerations for FFPE samples obtained for clinical evaluation. These may be associated with different contaminants or constraints in methodology for evaluation, and are discussed in more detail below $[37,38]$.

\section{Biomarkers by Type}

\section{Genomic biomarkers}

The European Medicines Agency, in concert with the International Council for Harmonisation of Technical Requirements for Pharmaceuticals for Human Use, has defined a genomic biomarker as "a measurable DNA and/or RNA characteristic that is an indicator of normal biologic processes, pathogenic processes, and/or response to therapeutic or other interventions"[39].

\section{Factors affecting genomic biomarkers}

Although DNA and RNA are generally reliable biomarkers, there are some commonly encountered situations in biospecimen collection that occur in clinical medicine that can affect nucleic acid quantity and quality and impact their accuracy as biomarkers. A few of these conditions are described here.
Pre-fixation time: Pre-fixation time is the duration of time between obtaining the biopsy or surgical specimen and its preservation. As the tissue samples removed are ischemic during this interval, several important biologic processes occur in the tissue that can affect nucleic acids. RNA, in particular, is susceptible to the effects of this "cold" (ie, $<37^{\circ} \mathrm{C}$ ) ischemia. Changes that are seen during cold ischemia include increased expression (quantity) of RNA molecules from hypoxia response genes (eg, hypoxia-inducible factor 1a [HIF1a]); digestion and loss of RNA molecules with short half-lives; and broad RNA degradation and reduction in quality, starting at about 5 to 6 hours at room temperature [40]. In general, the shorter the time from patient to preservation (preservative or freezing), the better.

Formalin: Formalin fixation is a common method used to preserve biological tissue samples that have been obtained surgically or by biopsy, and subsequent paraffin-embedding allows for the cutting of thin slices for histological examination. FFPE samples are abundant and represent the standard method of clinical tissue preservation in most hospitals. Formalin has several effects on DNA that affect DNA quality, including DNA denaturation and cross-linking with cytosine residues [41]. As a result of these and other effects on DNA, formalin induces artificial mutations at a rate of approximately 1 mutation per 500 base pairs. RNA shares these formalin effects, but it is also affected by formalin in other ways which impede reverse transcription $[41,42]$. Factors that increase the formalininduced artificial mutation rate include increasing formaldehyde concentration, increasing temperature, increasing duration of fixation, and decreasing $\mathrm{pH}$ [41].

Tissue nucleases: Deoxyribonucleases (DNases) and ribonucleases (RNases) are tissue nucleases that digest DNA and RNA, respectively. RNA molecules are particularly susceptible to degradation by RNases, and for this reason, RNase inhibition is part of most RNA extraction protocols. DNase is felt to be an important contributor to DNA degradation in FFPE tissue samples [43].

Storage conditions: The age of the FFPE sample and storage temperature can have an impact on nucleic acid quality [44]. In general, storage at $-20^{\circ} \mathrm{C}$ is better than room temperature, and shorter duration of storage is better.

\section{DNA}

DNA has many attributes that make it an excellent biomarker. First, DNA tends to be a very stable molecule-a biological requirement, as it directs the replication of all human cells-and is consequently 
affected less by environmental conditions than many other molecules. Second, many characteristics are measurable in DNA, including single-nucleotide variants (formerly single-nucleotide polymorphisms), variability of short repeated segments (eg, microsatellites), epigenetic modifications (eg, methylation), haplotypes, deletion mutations, insertion mutations, copy number variations, and cytogenetic variations (eg, translocations, duplications, deletions, or inversions).

One important distinction with DNA is the difference between germline changes and somatic changes. Germline DNA is the complement of genes that an individual is born with and can pass on to future progeny. Generally, blood leukocytes are used as the source for germline DNA, but there are scenarios (eg, leukemia) where this is not ideal, and buccal swabs, saliva, or other normal tissue are used. Most evidence suggests that buccal swabs and saliva yield similar DNA quality to blood leukocytes, although quantity is usually less $[45,46]$. Germline DNA alterations can inform the presence of an inherited tumour syndrome (eg, von Hippel-Lindau disease), a susceptibility to exposures (eg, glutathione-S-transferase [GSTM1] null and $\mathrm{N}$-acetyltransferase 2 [NAT2] slow acetylator increase the risk for bladder cancer), an ability to metabolize drugs, and a susceptibility to developing certain diseases or adverse events associated with treatment.

Somatic DNA refers to DNA collected from an affected tissue or organ, usually a tumour, and reflects a change that occurred in the DNA after conception. Somatic alterations are not passed on to children. Somatic alterations are useful for predicting responsiveness to treatment (eg, microsatellite instability and programmed death 1 ligand 1 [PD-L1] response), determining prognosis, and diagnosing the presence or absence of disease.

\section{RNA}

RNA is the transmitter of genetic information coded in the DNA and is therefore a significantly more dynamic molecule than DNA. RNA quantity and composition change significantly from tissue to tissue under normal physiologic conditions. Characteristics that are measured in RNA include sequences, splicing, expression levels, and subtype (eg, miRNA). As alluded to above, while RNA is a more responsive molecule and, perhaps, a better reflector of genetic activity within a particular tissue, it is also substantially less stable and is affected by a larger number of environmental conditions than DNA.

There are numerous types of RNA molecules and they are generally classified as the following: (a) those involved in protein synthesis, (b) those involved in RNA modification, and (c) those whose function is mainly regulatory [47]. A non-exhaustive summary of the main types of RNA is shown in Table 1.

\section{Protein}

Proteins are the workhorses of the cell and are often highly dysregulated in disease states. Proteins can be isolated from nearly all biofluids but, like all analytes, they are also subject to degradation and alteration. Human blood and urine contain proteases that cleave proteins into smaller peptides, which can be cleaved by peptidases into even smaller pieces [48]. Interestingly, the pattern of cleavage can be used as a signature to identify certain cancers [49]. Adding protease inhibitors to biospecimens can help reduce artifactual changes in proteins caused by enzymatic degradation, although these additions can also affect downstream applications.

Urine can be a particularly challenging source for protein biomarkers because of dramatic changes in $\mathrm{pH}$ (ranges from 4 to 8), the influence of hydration status on protein concentration, and proteolysis that occurs during storage in the bladder [50]. About 30\% of urinary proteins are derived from glomerular filtration and $70 \%$ from the renal tubules and urothelium, so the urine protein pool is a mix of systemic and local-regional sources [51].

Protein-based biomarkers have generally been focused on the quantification of a particular protein or isoform. However, assessment of post-translational modifications is also important. Post-translational modifications that can important to biomarkers include phosphorylation, methylation, glycosylation, ubiquitination, acetylation, and lipidation [52].

\section{Glycans}

The attachment of carbohydrates to molecules, such as proteins and lipids-a process known as glycosylationis common, occurring in $>50 \%$ of human proteins [53]. Several important glycoproteins have been found to be good biomarkers in urology, including $\alpha$-fetoprotein, prostate-specific antigen, and human chorionic gonadotropin. There are different forms of protein glycosylation, including $\mathrm{N}$-linked (glycan attached to the nitrogen of asparagine) and O-linked (glycan attached to the oxygen of threonine and serine). Tumours may show differences in the amount, size, and type of glycosylation when compared with normal tissue. For example, $\mathrm{N}$-linked glycans tend to become larger and more branched, whereas $\mathrm{O}$-linked glycans tend to be truncated and expose underlying peptide epitopes. Other glycans can be important biomarkers, too. For example, glycolipids (glycans bound to lipid molecules) and glycosaminoglycans (mucopolysaccharides) have been studied as biomarkers. 


\section{TABLE 1.}

\section{Main Types of RNA}

Protein Synthesis

\begin{tabular}{ll} 
Type & Function \\
\hline Messenger (mRNA) & $\begin{array}{l}\text { - Transcription of the information contained in DNA exons (recipe for a protein) } \\
\text { - Subject to alternative splicing, which creates different protein isoforms }\end{array}$ \\
\hline Ribosomal (rRNA) & $\begin{array}{l}\text { - Primary constituent of the ribosomes, where mRNA is translated into protein } \\
\text { - Most abundant RNA in cells (about } 80 \%)\end{array}$ \\
\hline Transfer (tRNA) & - Carries an amino acid matching the mRNA to the ribosome, required for translation
\end{tabular}

RNA modification

\begin{tabular}{|c|c|}
\hline Type & Function \\
\hline Small nuclear (snRNA) & - Processing and splicing of mRNA in the nuclear spliceosome \\
\hline $\begin{array}{l}\text { Small nucleolar } \\
\text { (snoRNA) }\end{array}$ & - Involved in methylation and pseudouridylation of rRNA and tRNA \\
\hline Ribonuclease P & - Riboenzyme (enzyme made of RNA) that cleaves RNA \\
\hline Ribonuclease MRP & - Riboenzyme that processes rRNA in the nucleus \\
\hline \multicolumn{2}{|l|}{ Regulatory } \\
\hline Type & Function \\
\hline Micro (miRNA) & - Single stranded RNA, 22 bp length, interferes with other RNAs \\
\hline $\begin{array}{l}\text { Small interfering } \\
\text { (siRNA) }\end{array}$ & - Double stranded RNA, 20-25 bp length, interferes with other RNAs \\
\hline $\begin{array}{l}\text { Long non-coding } \\
\text { (IncRNA) }\end{array}$ & - Single stranded RNA, >200 bp length, interferes with other RNAs \\
\hline Short hairpin (shRNA) & - Artificial RNA molecule designed to inhibit other RNAs, has a tight hairpin turn structure \\
\hline Antisense (asRNA) & - Single stranded RNA complementary to a mRNA to which it binds and inhibits \\
\hline
\end{tabular}

\section{Lipids}

Lipids are key molecules in cellular metabolism and are a critical structural component in the biological membranes that wrap all human cells. Lipids are different from other biomolecules in that they are soluble in organic solvents, which is an important processing step in lipid analysis and characterization [54]. Lipids are subdivided into 8 classes, each of which has had some biological role described in cancer biology: fatty acyls, glycerophospholipids, glycerolipids, sphingolipids, sterol lipids, prenol lipids, saccharolipids, and polyketides [55]. Mass spectroscopy and related techniques are the main tools used for profiling biological lipids.

\section{Imaging}

Although it may not seem intuitive, imaging can also serve as a biomarker [56,57]. Examples of widely available imaging-based biomarkers include basic radiological lesion characteristics (eg, size, shape, location), lesion density (computed tomography), lesion echogenicity (ultrasound), lesion signal intensity (magnetic resonance imaging), and contrast enhancement. The Response Evaluation Criteria In Solid Tumors (RECIST) criteria for evaluating tumour response to therapy is a radiological biomarker that is commonly used in clinical trials $[58,59]$. Functional molecular imaging has been further developed, whereby specific molecular features are studied using novel radiological ligands. For example, in positron emission tomography (PET) imaging, functional biomarkers are being explored to improve the detection of cancer, including, $18 \mathrm{~F}$-fluorodeoxyglucose (18F-FDG), carbon 11 choline (11C-choline), 68Gallium prostate-specific membrane antigen (68Ga-PSMA), and numerous others. 
In other cases, theranostic imaging is being pursued whereby a molecular target is imaged in a patient in vivo before the administration of a targeted agent against that molecular target [60].

\section{Pathology}

The histological evaluation of tissue samples (or blood smears) is not only a routine clinical component of cancer care but also an important source of clinical biomarkers. Many standard descriptors of tissue morphology can be quantified and used as biomarkers. Common examples in genitourinary oncology include tumour grade, presence of lymphovascular invasion, presence of mitoses, and histological tumour type and subtype. More recently, digital imaging has allowed for a new era of digital pathology, in which pattern recognition and artificial intelligence software tools can

\section{References}

1. Vandekerkhove G, Struss WJ, Annala M, et al. Circulating Tumor DNA Abundance and Potential Utility in De Novo Metastatic Prostate Cancer. Eur Urol. 2019;75(4):667-75. D0I: 10.1016/j. eururo.2018.12.042

2. How Kit A, Nielsen HM, Tost J. DNA methylation based biomarkers: practical considerations and applications. Biochimie. 2012;94(11):2314-37. DOl: 10.1016/j.biochi.2012.07.014

3. Loke SY, Lee ASG. The future of blood-based biomarkers for the early detection of breast cancer. Eur J Cancer. 2018;92:54-68. DOI: 10.1016/j.ejca.2017.12.025

4. Pietrowska M, Wlosowicz A, Gawin M, Widlak P. MS-Based Proteomic Analysis of serum and plasma: problem of high abundant components and lights and shadows of albumin removal. Adv Exp Med Biol. 2019;1073:57-76. DOI: 10.1007/978-3-030-12298-0_3

5. O'Connell GC, Treadway MB, Petrone AB, et al. Leukocyte dynamics influence reference gene stability in whole blood: data-driven qRT-PCR normalization is a robust alternative for measurement of transcriptional biomarkers. Lab Med. 2017;48(4):346-56. DOl: 10.1093/labmed/Imx035

6. Matomaki P, Kainulainen $H$, Kyrolainen $H$. Corrected whole blood biomarkers - the equation of Dill and Costill revisited. Physiol Rep. 2018;6(12):e13749. DOI: 10.14814/phy2.13749

7. Mitchell BL, Yasui Y, Li Cl, Fitzpatrick AL, Lampe PD. Impact of freeze-thaw cycles and storage time on plasma samples used in mass spectrometry based biomarker discovery projects. Cancer Inform. 2005;1:98-104.

8. Scaramuzzino DA, Schulte K, Mack BN, Soriano TF, Fritsche HA. Five-year stability study of free and total prostate-specific antigen concentrations in serum specimens collected and stored at -70 degrees C or less. Int J Biol Markers. 2007;22(3):206-13.

9. Elliot G. Preservation of biologics in a dry state: advances in isothermal vitrification technology. Cryobiology. 2013;67(3):428. DOI: https://doi.org/10.1016/j.cryobiol.2013.09.115 be used to characterize tissue sections with increasingly precise and reproducible methods [61,62]. It is highly likely that in the future digital pathology tools will form the backbone of the analysis of most tissue sections.

\section{Conclusions}

Biomarkers can be obtained and characterized from a highly diverse set of biological sources of measurement. There is no clear optimal biomarker, and each has inherent strengths and flaws. The future will likely consist of a collation of large networks of biomarkers that are merged computationally to provide a consensus picture of the pathological process that is occurring in the patient. This will undoubtedly require new informatic and artificial intelligence tools but will also lead to a new era of precision medicine.

10. Kluge JA, Li AB, Kahn BT, Michaud DS, Omenetto FG, Kaplan DL. Silk-based blood stabilization for diagnostics. Proc Natl Acad Sci U S A. 2016;113(21):5892-7. D0I: 10.1073/pnas.1602493113

11. Jackson DH, Banks RE. Banking of clinical samples for proteomic biomarker studies: a consideration of logistical issues with a focus on pre-analytical variation. Proteomics Clin Appl. 2010;4(3):250-70. DOI: 10.1002/prca.200900220

12. Dittadi R, Fabricio ASC, Rainato G, et al. Preanalytical stability of [-2]proPSA in whole blood stored at room temperature before separation of serum and plasma: implications to Phi determination. Clin Chem Lab Med. 2019;57(4):521-31. D0I: 10.1515/cclm-2018-0596

13. Cantiello F, Russo GI, Vartolomei MD, et al. Systemic inflammatory markers and oncologic outcomes in patients with high-risk non-muscle-invasive urothelial bladder cancer. Eur Urol Oncol. 2018;1(5):403-10. DOI: 10.1016/j.euo.2018.06.006

14. Bartlett EK, Flynn JR, Panageas KS, et al. High neutrophil-tolymphocyte ratio (NLR) is associated with treatment failure and death in patients who have melanoma treated with PD-1 inhibitor monotherapy. Cancer. 2020;126(1):76-85. DOI: 10.1002/cncr.32506

15. Okita K, Hatakeyama S, Tanaka T, et al. Impact of disagreement between two risk group models on prognosis in patients with metastatic renal-cell carcinoma. Clin Genitourin Cancer. 2019;17(3):e440-e6. D0I: 10.1016/j.clgc.2019.01.006

16. Zhao L, He R, Long $H$, et al. Late-stage tumors induce anemia and immunosuppressive extramedullary erythroid progenitor cells. Nat Med. 2018;24(10):1536-44. DOI: 10.1038/s41591-018-0205-5

17. Atkins CG, Buckley K, Blades MW, Turner RFB. Raman Spectroscopy of blood and blood components. App/ Spectrosc. 2017;71(5):767-93. DOI: $10.1177 / 0003702816686593$

18. Riedhammer C, Halbritter D, Weissert R. Peripheral blood mononuclear cells: isolation, freezing, thawing, and culture. Methods Mol Biol. 2016;1304:53-61. D0l: 10.1007/7651_2014_99 
19. Klein A, Ramcharitar S, Christeff N, Nisbett-Brown E, Nunez E, Malkin A. Effect of anticoagulants in vitro on the viability of lymphocytes and content of free fatty acids in plasma. In Vitro Cell Dev Biol. 1991;27a(4):307-11. D0I: 10.1007/bf02630908

20. Buhl T, Legler TJ, Rosenberger A, Schardt A, Schon MP, Haenssle HA. Controlled-rate freezer cryopreservation of highly concentrated peripheral blood mononuclear cells results in higher cell yields and superior autologous T-cell stimulation for dendritic cell-based immunotherapy. Cancer Immunol Immunother. 2012;61(11):2021-31. DOI: $10.1007 / \mathrm{s} 00262-012-1262-0$

21. McKinnon KM. Flow cytometry: an overview. Curr Protoc Immunol. 2018;120:5.1.-5.1.11. D0I: 10.1002/cpim.40

22. Rodrigues D, Jeronimo $C$, Henrique $R$, et al. Biomarkers in bladder cancer: a metabolomic approach using in vitro and ex vivo model systems. Int J Cancer. 2016;139(2):256-68. DOI: 10.1002/ijc.30016

23. Wang X, Gu H, Palma-Duran SA, et al. Influence of storage conditions and preservatives on metabolite fingerprints in urine. Metabolites. 2019;9(10):203.DOI: 10.3390/metabo9100203

24. Merchant ML, Rood IM, Deegens JKJ, Klein JB. Isolation and characterization of urinary extracellular vesicles: implications for biomarker discovery. Nat Rev Nephrol. 2017;13(12):731-49. DOI: 10.1038/nrneph.2017.148

25. De Palma G, Di Lorenzo VF, Krol S, Paradiso AV. Urinary exosomal shuttle RNA: promising cancer diagnosis biomarkers of lower urinary tract. Int J Biol Markers. 2019;34(2):101-7. DOI: $10.1177 / 1724600819827023$

26. Roberts MJ, Richards RS, Gardiner RA, Selth LA. Seminal fluid: a useful source of prostate cancer biomarkers? Biomark Med. 2015;9(2):77-80. DOI: 10.2217/bmm.14.110

27. Etheridge T, Straus J, Ritter MA, Jarrard DF, Huang W. Semen AMACR protein as a novel method for detecting prostate cancer. Urol Oncol. 2018;36(12):532.e1-.e7. D0l: 10.1016/j. urolonc.2018.09.010

28. Ponti G, Maccaferri M, Mandrioli M, et al. Seminal cell-free DNA assessment as a novel prostate cancer biomarker. Pathol Oncol Res. 2018;24(4):941-5. DOI: 10.1007/s12253-018-0416-6

29. Ploussard G, de la Taille A. The role of prostate cancer antigen 3 (PCA3) in prostate cancer detection. Expert Rev Anticancer Ther. 2018;18(10):1013-20. DOI: 10.1080/14737140.2018.1502086

30. GoessI C, Muller M, Heicappell R, Krause H, Miller K. DNA-based detection of prostate cancer in blood, urine, and ejaculates. Ann N Y Acad Sci. 2001;945:51-8. D0I: 10.1111/j.1749-6632.2001.tb03863.x

31. Moschini M, Spahn M, Mattei A, Cheville J, Karnes RJ. Incorporation of tissue-based genomic biomarkers into localized prostate cancer clinics. BMC Med. 2016;14:67. D0I: 10.1186/s12916-016-0613-7

32. Tao DL, Bailey S, Beer TM, et al. Molecular Testing in Patients With Castration-Resistant Prostate Cancer and Its Impact on Clinical Decision Making. JCO Precis Oncol. 2017;1. DOI: 10.1200/ po.16.00067
33. Wahlin S, Nodin B, Leandersson K, Boman K, Jirstrom K. Clinical impact of $T$ cells, B cells and the PD-1/PD-L1 pathway in muscle invasive bladder cancer: a comparative study of transurethral resection and cystectomy specimens. Oncoimmunology. 2019;8(11):e1644108. D0I: 10.1080/2162402x.2019.1644108

34. Xiong Y, Liu L, Xia Y, et al. Tumor infiltrating mast cells determine oncogenic HIF-2alpha-conferred immune evasion in clear cell renal cell carcinoma. Cancer Immunol Immunother. 2019;68(5):731-41. DOI: 10.1007/s00262-019-02314-y

35. Hendry S, Salgado R, Gevaert T, et al. Assessing tumor-infiltrating Iymphocytes in solid tumors: a practical review for pathologists and proposal for a standardized method from the International ImmunoOncology Biomarkers Working Group: Part 2: TILs in melanoma, gastrointestinal tract carcinomas, non-small cell lung carcinoma and mesothelioma, endometrial and ovarian carcinomas, Squamous Cell Carcinoma of the Head and Neck, Genitourinary Carcinomas, and Primary Brain Tumors. Adv Anat Pathol. 2017;24(6):311-35. D0I: 10.1097/pap.0000000000000161

36. Cole LM, Clench MR, Francese S. Sample treatment for tissue proteomics in cancer, toxicology, and forensics. Adv Exp Med Biol. 2019;1073:77-123. DOI: 10.1007/978-3-030-12298-0_4

37. Jacobs S. Sample processing considerations for detecting copy number changes in formalin-fixed, paraffin-embedded tissues. Cold Spring Harb Protoc. 2012;2012(11):1195-202. DOI: 10.1101/ pdb.ip071753

38. Jacobs S. Data analysis considerations for detecting copy number changes in formalin-fixed, paraffin-embedded tissues. Cold Spring Harb Protoc. 2012;2012(11):1203-9. DOl: 10.1101/pdb.ip071761

39. European Medicines Agency. ICH Topic E15. Definitions for genomic biomarkers, pharmacogenomics, pharmacogenetics, genomic data and sample coding categories2007. Available from: https:// .ema.europa.eu/en/documents/scientific-guideline/ich-e-15establish-definitions-genomic-biomarkers-pharmacogenomicspharmacogenetics-genomic-data_en.pdf.Accessed: August 10, 2020.

40. Grizzle WE, Otali D, Sexton KC, Atherton DS. Effects of cold ischemia on gene expression: a review and commentary. Biopreserv Biobank. 2016;14(6):548-58. DOl: 10.1089/bio.2016.0013

41. Srinivasan M, Sedmak D, Jewell S. Effect of fixatives and tissue processing on the content and integrity of nucleic acids. Am J Pathol. 2002;161(6):1961-71. DOI: 10.1016/s0002-9440(10)64472-0.

42. Evers DL, Fowler CB, Cunningham BR, Mason JT, O'Leary TJ. The effect of formaldehyde fixation on RNA: optimization of formaldehyde adduct removal. J Mol Diagn. 2011;13(3):282-8. D0I: 10.1016/j.jmoldx.2011.01.010

43. Tokuda Y, Nakamura T, Satonaka K, et al. Fundamental study on the mechanism of DNA degradation in tissues fixed in formaldehyde. J Clin Pathol. 1990;43(9):748-51. DOl: 10.1136/jcp.43.9.748 
44. Groelz D, Viertler C, Pabst D, Dettmann N, Zatloukal K. Impact of storage conditions on the quality of nucleic acids in paraffin embedded tissues. PLoS One. 2018;13(9):e0203608. D0I: 10.1371/ journal.pone.0203608

45. King IB, Satia-Abouta J, Thornquist MD, et al. Buccal cell DNA yield, quality, and collection costs: comparison of methods for large-scale studies. Cancer Epidemiol Biomarkers Prev. 2002;11(10 Pt 1):1130-3.

46. Hansen TV, Simonsen MK, Nielsen FC, Hundrup YA. Collection of blood, saliva, and buccal cell samples in a pilot study on the Danish nurse cohort: comparison of the response rate and quality of genomic DNA. Cancer Epidemiol Biomarkers Prev. 2007;16(10):20726. DOI: 10.1158/1055-9965.Epi-07-0611

47. Xi X, Li T, Huang $Y$, et al. RNA biomarkers: frontier of precision medicine for cancer. Noncoding RNA. 2017;3(1):9. DOI: 10.3390/ ncrna3010009

48. Kushnir MM. Are samples in your freezer still good for biomarker discovery? Am J Clin Pathol. 2013;140(3):287-8. D0l: 10.1309/ ajcpfzyy7bbkk9je

49. Villanueva J, Shaffer DR, Philip J, Chaparro CA, Erdjument-Bromage $H$, Olshen $A B$, et al. Differential exoprotease activities confer tumorspecific serum peptidome patterns. J Clin Invest. 2006;116(1):27184. DOI: $10.1172 /$ jci26022

50. Thomas CE, Sexton W, Benson K, Sutphen R, Koomen J. Urine collection and processing for protein biomarker discovery and quantification. Cancer Epidemiol Biomarkers Prev. 2010;19(4):9539. DOI: 10.1158/1055-9965.Epi-10-0069

51. Harpole M, Davis J, Espina V. Current state of the art for enhancing urine biomarker discovery. Expert Rev Proteomics. 2016;13(6):609-26. DOI: 10.1080/14789450.2016.1190651

52. Khoury GA, Baliban RC, Floudas CA. Proteome-wide post-translational modification statistics: frequency analysis and curation of the swiss-prot database. Sci Rep. 2011;1. D0I: 10.1038/ srep00090.
53. Kailemia MJ, Park D, Lebrilla CB. Glycans and glycoproteins as specific biomarkers for cancer. Anal Bioanal Chem. 2017;409(2):395-410. DOI: 10.1007/s00216-016-9880-6

54. Zhao YY, Cheng XL, Lin RC. Lipidomics applications for discovering biomarkers of diseases in clinical chemistry. Int Rev Cell Mol Biol. 2014;313:1-26. DOI: 10.1016/b978-0-12-800177-6.00001-3

55. Stephenson DJ, Hoeferlin LA, Chalfant CE. Lipidomics in translational research and the clinical significance of lipid-based biomarkers. Trans/ Res. 2017:189:13-29. D0I: 10.1016/j. trsl.2017.06.006

56. White paper on imaging biomarkers. Insights Imaging. 2010;1(2): 42-5. DOI: 10.1007/s13244-010-0025-8

57. Medical imaging in personalised medicine: a white paper of the research committee of the European Society of Radiology (ESR). Insights Imaging. 2011;2(6):621-30. DOI: 10.1007/s13244-011-0125-0

58. Schwartz LH, Litiere S, de Vries E, et al. RECIST 1.1-Update and clarification: From the RECIST committee. Eur J Cancer. 2016;62:132-7 DOI: 10.1016/j.ejca.2016.03.081.

59. Seymour L, Bogaerts J, Perrone A, et al. iRECIST: guidelines for response criteria for use in trials testing immunotherapeutics. Lancet Oncol. 2017;18(3):e143-e52. D0I: 10.1016/s1470-2045(17)30074-8

60. Turner $\mathrm{JH}$. An introduction to the clinical practice of theranostics in oncology. Br J Radiol. 2018;91(1091):20180440. DOI: 10.1259/ bjr.20180440

61. Janowczyk A, Madabhushi A. Deep learning for digital pathology image analysis: A comprehensive tutorial with selected use cases. J Pathol Inform. 2016;7:29. DOI: 10.4103/2153-3539.186902

62. Janowczyk A, Zuo R, Gilmore H, Feldman M, Madabhushi A. Histo0C: an open-source quality control tool for digital pathology slides. JCO Cli n Cancer Inform. 2019:3:1-7. DOI: 10.1200/cci.18.00157 